\title{
Spinal cord regeneration in rats made immunologically unresponsive to CNS antigens
}

\author{
E.R. FER I N G A, K.R. NELSON, H.L. VA H LS I N G, \\ A N D R. C. D A USER \\ From the Departments of Neurology and Pathology, The Veterans Administration and University \\ of Michigan Medical Centers, Ann Arbor, Michigan, USA
}

SUMMARY Seventy days after complete spinal cord transection, both treated and untreated rats showed evidence that some corticospinal axons had regenerated. Rats made immunologically unresponsive to CNS tissue showed no increase in corticospinal regeneration as measured by orthograde axoplasmic flow of tritiated proline or retrograde axoplasmic labelling with horseradish peroxidase. However, treated rats did demonstrate electrophysiological evidence of regeneration of long ascending sensory pathways. Tolerant animals additionally treated with cyclophosphamide showed corticospinal axonal regeneration by tritiated proline transport and electrophysiological techniques and also showed electrophysiological evidence of ascending sensory tract regeneration.

In several previous experiments, we have shown that treatments designed to suppress the immune response have resulted in increased regeneration of corticospinal neurones or other long tracts in the rat spinal cord (Feringa et al., 1973, 1974, $1975,1977,1978)$. Our most successful treatments have been in animals which were treated 48 hours after spinal cord transection with a single dose of cyclophosphamide. In such animals, we have shown: (1) increased regeneration of corticospinal tracts, by tritiated proline transport techniques (Feringa et al., 1977); (2) the presence of descending motor fibres which cross at the point of transection, by Fink-Heimer/Nauta techniques (Feringa et al., 1978); and (3) evidence for conduction of an action potential by the long tracts of the cord across the site of transection, by the evoked motor response technique (Feringa et al., 1973, 1974, 1975, 1977). A few animals in each group showed significant regeneration, and careful statistical evaluation demonstrated a difference between immunosuppressed and control animals.

There have been indications that immunosuppressive treatments other than cyclophosphamide also enhance central nervous system (CNS) regeneration, but the data were far from

Address for reprint requests: Dr Earl R. Feringa, Chief, Neurology Service (127), Veterans Administration Medical Center, 2215 Fuller Road, Ann Arbor, Michigan 48105, USA.

Accepted 13 January 1979 conclusive (Feringa et al., 1973). The effects of cyclosphosphamide are varied and by no means limited to a specific anti-immune activity. While the timing of the most successful treatment with cyclophosphamide (injection 48 hours after spinal cord transection) is compatible with the idea that cyclophosphamide is working in its immunosuppressive role (Paterson and Hanson, 1969), it is quite possible that our positive responses were due to a more nonspecific drug action. The timing of such a nonspecific action might also be critical and yield most effective results if administered at the same 48 hour interval after transection (Feringa et al., 1977; Willenborg et al., 1977; Feringa et al., 1978).

Because of this uncertainty we devised this experiment to determine if induced immunological unresponsiveness to CNS antigens, a treatment whose only known effect is to blunt the immune response, would enhance regeneration. We also used induced unresponsiveness in conjunction with our sucessful cyclophosphamide treatment to learn what effect the combined treatment might have (Weigle, 1973).

\section{Material and methods}

ANIMALS

All animals used in this experiment were isogeneic female rats inbred for at least $\mathbf{8 1}$ generations. Only 
females were used because of the urological complications in paraplegic male animals.

Four groups were studied. Group $A$ was a control group which consisted of 18 female rats which received no special pre- or postspinal cord transection treatment. Spinal cord transections in this group, and in groups $B$ and $C$ were performed on adult animals, six weeks of age, 125 grams weight. Group $B$ was a group of 11 female rats in which we induced immunological unresponsiveness to CNS antigens. They were injected intraperitoneally with $75 \mathrm{mg}$ of spinal cord emulsified in saline on the first day of life and every week until the time of spinal cord transection. Emulsified spinal cord was obtained from other female isogeneic rats. Group $C$ was a group of 11 female animals who were also treated with injections of emulsified spinal cord on the first day of life and weekly until the time of spinal cord transection. These animals were additionally treated with a single injection of $75 \mathrm{mg} / \mathrm{kg}$ cyclophosphamide intraperitoneally 48 hours after spinal cord transection. Group $D$ was a group of nine female animals designated as a short-term control group. Spinal cord transections were performed at 10-13 weeks of age in this group only. They were tested separately five weeks after spinal cord transection to define the specificity and error of the tests of axonal transport of tritiated proline.

The flow of treatments and testing is shown in Table 1.

\section{SURGICAL TECHNIQUE}

With the aid of a binocular surgical microscope, a midthoracic laminectomy was performed on rats anaesthetised with ether. The spinal cord was transected completely at $\mathrm{T} 7$ or $\mathrm{T} 8$ segments. In each case the completeness of the transection was confirmed by a co-worker. Subsequent histological evaluation confirmed that these transections had severed the entire spinal cord. Postoperatively the bladder was emptied using the method of Credé every eight hours until automatic bladder function was restored (usually two to three weeks).

\section{EVALUATION PROCEDURES}

\section{Electrophysiological evaluation}

Seventy days after spinal cord transection, the cervical spinal cord was exposed in anaesthetised animals of groups A, B, and C. Each animal was immobilised by an intraperitoneal injection of 0.16 to $0.40 \mathrm{mg}$ pancuronium bromide. A concentric bipolar stimulation needle electrode was inserted into the posterior columns of the cervical spinal cord in the area of the corticospinal tracts. Evoked potentials were sought by bipolar recording from individual dorsal and ventral rootlets exposed as they left the lumbosacral cord, well below the point of spinal cord transection. For each animal eight dorsal and eight ventral rootlets distal to the transection site were studied. For each individual rootlet, 128 stimulus-responsive recordings were averaged by computer. The results were displayed on a cathode-ray oscilloscope and photographed. The photographs were evaluated by all investigators in a blind fashion for evidence of spinal axonal regeneration. The electrophysiological technique is illustrated in Fig. 1.

\section{Radioisotope studies}

Each animal was studied for transport of tritiated proline by axonal flow down the corticospinal tract across the site of the spinal cord transection. Twenty-one days before the planned date of electrophysiological testing and/or sacrifice, the sensorimotor cortex of each animal was injected with tritiated proline. The sensorimotor cortex is located approximately $3 \mathrm{~mm}$ lateral to the sagittal suture and $2 \mathrm{~mm}$ caudal to the coronal suture (Hicks and D'Amato, 1975). Using a dental drill under a constant stream of saline to minimise heat effects, a hole was made in the skull at this area down to, but not through, the dura mater. A micromanipulator and a Hamilton syringe with a 32 gauge short bevel needle was used to inject $2.5 \mu \mathrm{l}$ of a suspension containing 50 microcuries of tritiated proline into the cerebral cortex $1 \mathrm{~mm}$ below the cortical surface. The proline was injected very slowly over a period of 30-60 seconds.

Table 1 Age of treatment groups at various stages of the experiment

\begin{tabular}{|c|c|c|c|c|}
\hline \multirow[t]{2}{*}{ Event } & \multicolumn{4}{|c|}{ Treatment Group } \\
\hline & $A$ & $B$ & $C$ & $D$ \\
\hline Weekly injection of spinal cord antigen & & $1-5 \mathrm{wk}$ & $1-5 \mathrm{wk}$ & \\
\hline Complete spinal cord transection & $6 \mathrm{wk}$ & $6 \mathrm{wk}$ & $6 \mathrm{wk}$ & $10-13 w k$ \\
\hline Cyclophosphamide injection $75 \mathrm{mg} / \mathrm{kg}$ & & & $6 w k+2$ & \\
\hline Tritiated proline injected into sensorimotor cortex & $13 \mathrm{wk}$ & $13 w k$ & 13 wk & $12-15 \mathrm{wk}$ \\
\hline Electrophysiology followed by HRP insertion & $16 \mathrm{wk}$ & 16 wk & $16 \mathrm{wk}$ & \\
\hline Perfusion & 16 wk $3 \mathrm{~d}$ & 16 wk $3 \mathrm{~d}$ & 16 wk $3 \mathrm{~d}$ & $15-18 \mathrm{wk}$ \\
\hline
\end{tabular}




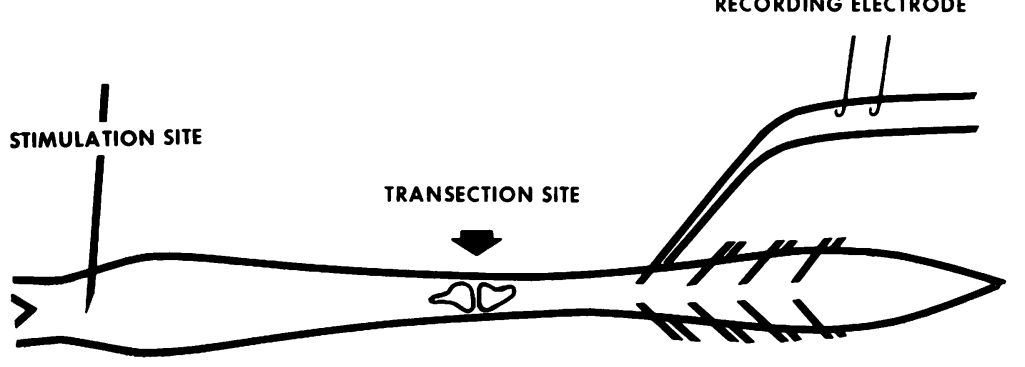

Fig. 1 Line drawing of the spinal cord from the obex to the cauda equina illustrating the electrophysiological technique. The spinal cord transection was at $T 7$ or $T 8$ segment. Stimulation was applied at $C 2$ or $C 3$ segment. Responses were detected from individual dorsal and ventral roots of the lumbosacral enlargement.
The needle was then allowed to remain in place for another 60 seconds before it was withdrawn. A similar injection was then performed into the contralateral sensorimotor cortex.

On completion of electrical testing the animals were allowed to live for three days, and were then perfused with $1 \%$ paraformaldehyde, $1.25 \%$ gluteraldehyde in $\mathbf{p H} 7.2$ phosphate buffer (LaVail, 1975), and the spinal cord was exposed. Identical $15 \mathrm{~mm}$ measured lengths of spinal cord were taken from the cervical region above the site of spinal cord transection and from the lumbosacral region below the transection (see Fig. 2). Each segment of spinal cord was dissolved and scintillation counted for tritium. The disintegrations per minute (DPM) in the proximal segment measured the success with which we had labelled the corticospinal tracts. Disintegrations per minute in the distal segment expressed as a percentage of the label present in the proximal segment is a measure of corticospinal axonal regeneration.

\section{Horseradish peroxidase studies}

At the completion of electrical testing in the lumbosacral enlargement, a small V-shaped cut was made in the area of the dorsal white columns in which the corticospinal tracts run in the rat. A small $(0.5 \mathrm{mg})$ pellet of horseradish peroxidase (HRP) crystals was inserted so that the crystals were in direct contact with the corticospinal tract fibres. The wound was closed and the animal allowed to recover from anaesthesia and neuromuscular blockage. Three days later, anaesthetised rats were perfused, first with saline then with
$40 \mathrm{ml}$ of $6 \%$ dextran, and finally with $40 \mathrm{ml}$ of paraformaldehyde, gluteraldehyde fixative.

The entire brain was removed and fixed for four hours at $4^{\circ} \mathrm{C}$ in fixative. Blocks cut to include the sensorimotor cortex were transferred to cold $5 \%$ sucrose in phosphate buffer $(\mathrm{pH} 7.2)$ and held overnight at $4^{\circ} \mathrm{C}$. The next day $40 \mu \mathrm{m}$ frozen sections were cut and collected in cold sucrose buffer. Each section was incubated for 10 to 15 minutes in $5 \mathrm{mg}$ of $3.3^{\prime}$ diaminobenzidine dissolved in $10 \mathrm{ml}$ of $0.1 \mathrm{M}$ phosphate buffer (pH 6.0) with $0.3 \mathrm{ml}$ of $0.3 \% \quad \mathrm{H}_{2} \mathrm{O}_{2}$. Stained sections were mounted on glass slides. Thirty sections of cortex from each animal were evaluated blindly. Each section was searched carefully using both bright and dark field optics for labelled cortical neurones.

\section{Results}

Four animals demonstrated electrophysiological evidence of regeneration of long spinal cord tracts (Table 2). In each animal, a stimulus applied to the high cervical spinal cord resulted in a propagated impulse detected in one or more dorsal roots. In one animal a response was also found in one ventral root. Two animals were in the group which had been made immunotolerant before spinal cord transection (group B). Two were in the group which had both been made immunotolerant to CNS antigen and had immunological response suppressed by subsequent postoperative cyclophosphamide treatment (group C). The one animal showing a response in both dorsal and ventral

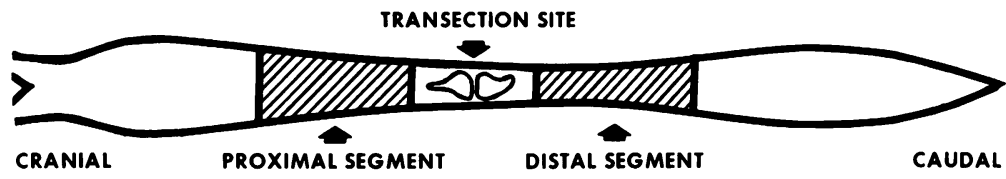

Fig. 2 This line drawing shows the location of spinal cord segments which were used to measure tritiated proline transported by corticospinal axons. Segments were taken $10 \mathrm{~mm}$ either side of the transection site so that the cysts at the transection site were not included in the segment counted. 
Table 2 Results of electrophysiological testing

\begin{tabular}{llll}
\hline Group & $\begin{array}{l}\text { Number of rats } \\
\text { showing } \\
\text { transmission }\end{array}$ & $\begin{array}{l}\text { Number of rats } \\
\text { not showing } \\
\text { transmission }\end{array}$ & $P$ \\
\hline A (control) & 0 & 17 & 0.046 \\
B (immunotolerant) & 2 & 9 & 0.039 \\
$\begin{array}{l}\text { C (immunotolerant } \\
\text { and suppressed) }\end{array}$ & 2 & 8 & \\
\hline
\end{tabular}

roots was also in group C. Statistical analysis of the difference between the test groups and the control animals indicated that both group $B$ and group $C$ showed more regeneration than the control animals.

The results of our studies of tritiated proline transported by axonal flow are shown in Table 3. In the group A (control) animals an average $6.9 \%$ of the radioactivity located in the cervical region could be found in the distal segment of spinal cord. In the immunotolerant animals, $6.6 \%$ was detected in the distal cord. These numbers are not significantly different. However, in the group which was both made tolerant to CNS antigens and received cyclophosphamide postoperative treatment, $12.7 \%$ of the radioactivity located in the proximal segment was also found in the distal segment. This group is significantly different from the control group.

In the short-term control group, only $2.0 \%$ of the radioactivity found in the cervical region was found in the distal segment. The results of all animals were grouped very tightly together in the range of 1.1 to $4.2 \%$. This measures the amount of radioactivity that reaches the distal cord by routes other than axonal transport. It is, therefore, a measure of the intrinsic error of this method of evaluation.

None of the cortical slides showed any HRP labelled cells. Using the identical procedure in normal (nontransected) rats of the same strain and age, we invariably achieved good staining of many cortical neurones.

Table 3 Transport of tritiated proline by regenerated corticospinal axons

\begin{tabular}{|c|c|c|c|c|}
\hline Group & Number & $\begin{array}{l}\text { Mean value } \\
\text { DPM distall } \\
\text { DPM } \\
\text { proximal }\end{array}$ & $\begin{array}{l}P \\
\text { (compared } \\
\text { to group } \\
\text { A) }\end{array}$ & $\begin{array}{l}P \\
\text { (co npared } \\
\text { to group } \\
\text { D) }\end{array}$ \\
\hline $\begin{array}{l}\text { A (control) } \\
\text { B (immunotolerant) } \\
\text { C (immunotolerant } \\
\text { and suppressed) } \\
\text { D (short-term } \\
\quad \text { control) }\end{array}$ & $\begin{array}{r}18 \\
11 \\
11 \\
9\end{array}$ & $\begin{array}{l}0.069 \\
0.066 \\
0.127 \\
0.020\end{array}$ & $\begin{array}{l}0.392 \\
0.037 \\
0.001\end{array}$ & $\begin{array}{l}0.001 \\
0.015 \\
\\
0.019\end{array}$ \\
\hline
\end{tabular}

$\mathrm{DPM}=$ disintegrations per minute (scintillation count)

\section{Discussion}

\section{SELECTION OF TREATMENT GROUPS}

A successful protocol for inducing and maintaining immunological unresponsiveness to CNS antigens has yet to be developed (Weigle, 1972, 1973). Previous efforts to facilitate regeneration by inducing unresponsiveness have given equivocal results (Feringa et al., 1973, 1974, 1977, 1978). In each experiment we attempted to create tolerance with a single injection of spinal cord emulsion on the first day of life. Multiple injections of antigen begun during the neonatal period are more effective than a single injection (Paterson, 1958; Smith and Bridges, 1958; Eitzman and Smith, 1959; Weigle, 1973). Therefore, in this experiment we treated animals on the first day of life and weekly with intraperitoneal injections of $75 \mathrm{mg}$ isogeneic emulsified spinal cord in an effort to achieve a greater degree of immunological unresponsiveness than we have achieved in earlier experiments.

Cyclophosphamide in a single large dose given to rats two, three, or four days after inoculation with a homogenate of pig spinal cord and Freund's complete adjuvant completely inhibited clinical signs of experimental allergic encephalomyelitis (Paterson and Hanson, 1969). Experiments in our laboratory show that a similar single large dose treatment 24 or 48 hours after spinal cord transection in some way facilitates CNS regeneration (Feringa et al., 1974, 1975, 1977, 1978). Cyclophosphamide is known not to interfere with the induction of the immunological unresponsive state (Claman and Bronsky, 1965). Group C was selected to test the effects of combined induced immunological unresponsiveness and cyclophosphamide drug treatment.

\section{END POINTS}

The electrophysiological test detects regenerated corticospinal axons and can also detect regenerated long ascending sensory pathways which are stimulated antidromically. Such sensory axons, however, could be detected only if they regenerated over many spinal segments from the T7 or T8 transection site to the $\mathrm{C} 1$ or $\mathrm{C} 2$ stimulation site (Fig. 1). They would not be detected by the radioisotope tests. Impulses recorded from ventral roots are probably due to regenerated corticospinal tract axons, although there is recent evidence that ventral roots contain some sensory fibres (Clifton et al., 1976). Stimulus response latency was consistent with a conduction velocity of $10-20 \mathrm{~m} / \mathrm{s}$. This result is inconsistent with conduction via the sympathetic chain where conduction velocities are much slower $(1.69-2.3 \mathrm{~m} / \mathrm{s})$ (Hopkins and Lambert, 1972). 
The tritiated proline method is more sensitive for detection of regenerated corticospinal axons than the electrophysiological test, because it labels any corticospinal axon whereas the electrophysiological method detects only those axons which regenerate and form a synapse on a lower motor neurone whose axon was near our recording electrode.

Tritiated proline injected into the sensorimotor cortex in the manner described does not cause observable disruption of anatomical or functional activity of the cortex. The proline is rapidly absorbed by the cells in the cortex and is transported in an orthograde direction down the corticospinal tracts (Vahlsing et al., 1978). Autoradiograms of normal rat spinal cord after such treatment and autoradiograms of the cervical cord in these experimental animals demonstrate that the radioactive label is sharply restricted to the corticospinal tracts as they lie in the most ventral portion of the dorsal columns (Feringa et al., 1977). There is no evidence that radioactivity spreads significantly outside of the injected area of the brain except by way of orthograde axonal transport. There is no evidence in our animals to support the idea that this label is transmitted across synaptic junctions in the corticospinal system of the rat CNS.

Because the corticospinal tract runs almost exclusively in a linear fashion, equal lengths of cord are more appropriate for measuring corticospinal tracts than are equal weights of the cord segments. The spinal cord weight would vary with the amount of grey matter and other long tracts and not vary as directly with the amount of corticospinal tract contained within the segment.

Although autoradiograms indicated that all detectable radioactivity (above background) in the spinal cord was restricted to the corticospinal tract (Feringa et al., 1975) it seemed likely that a small amount of radioactivity might be dispersed more generally throughout the animal, having been ab- sorbed by the blood vessels in the cerebral cortex rather than by adjacent cortical neurones. To control for this we selected a group of animals whose age at the time of spinal cord transection varied from 10-13 weeks. Two weeks were allowed between spinal cord transection and intracortical injection with tritiated proline. After permitting the uniform three weeks for slow axoplasmic flow to label the corticospinal tracts, we studied these animals five weeks after spinal cord transection. The segments used for scintillation counting procedures were taken approximately $10 \mathrm{~mm}$ away from the site of spinal cord transection and, therefore, it would be unlikely that any axons which had begun to regenerate would have successfully crossed the site of injury and reached the distal segment in five weeks.

While electrophysiological data indicated improved regeneration in both treated groups, and while radioisotope data confirmed increased regeneration of corticospinal axons in group C, HRP implanted in the lumbar cord did not label cortical neurones in the sensorimotor cortex. Several explanations are possible. Perhaps immature regenerated axons (unmyelinated as yet?) are not able to transport HRP well enough to label corticalo neurones. Alternatively, the trauma associated with electrophysiological testing, anaesthesia, neuromuscular blockade and so on, might be expected to thwart successful use of this capricious staining technique. Because tritiated proline injected into the sensorimotor cortex was transported orthogradely by regenerated axons, our search for HRP labelled axons was limited to the sensorimotor cortex. Perhaps a much more extensive search of cortical and subcortical neurones would have detected labelled cells, but such a study was beyond the resources of our staff.

CONCURRENCE OF DATA

Figure 3 includes both the electrophysiological data and the range of the tritiated proline results.

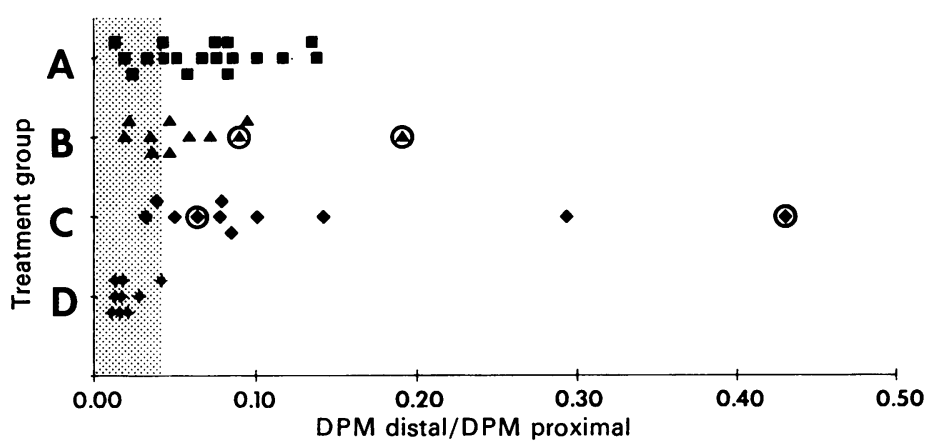

Fig. 3 Display of the values representing transport of tritiated proline by corticospinal axons in each animal. Circled symbols identify the animals which also showed electrophysiological evidence of regenerated axons. Shaded area represents the range of values for the short-term control series (see text). DPM= disintegrations per minute (scintillation count). 
Comparison of the regeneration measured by the tritiated proline transport data and the range of values in each group suggests that some corticospinal tract regeneration occurred in all groups by 70 days after spinal cord transection. It is conceivable that a few corticospinal axons do get across the gap in all animals by this period of time, but only those animals with a rather large amount of corticospinal tract regeneration have a probability of making a synapse which might be picked up by our electrophysiological testing. This finding is in agreement with data from two previous studies (Feringa et al., 1977, 1978).

All animals in which electrophysiological conduction was demonstrated (those circled in the graph in Fig. 3) showed a greater amount of axonal transported tritiated proline than did any of the short-term control animals. The animal which conducted the most tritiated proline was the one in which a response was detected in both dorsal and ventral roots. This was the only animal which conducted an impulse that was detected from a ventral root, indicating that the regenerated fibre was probably corticospinal in origin.

In one group $\mathrm{C}$ animal identified as a nonresponder, $29 \%$ of the radioactivity in the proximal segment was found in the distal segment (Fig. 3). Both a dorsal and a ventral root of this animal showed questionable electrophysiological responses, but she was designated a "nonresponder" after considerable debate, This was the only animal whose electrophysiological data would be more accurately termed equivocal.

\section{CONCLUSION}

Since those animals in group B were treated only with weekly injections of CNS antigen to produce an immunological unresponsive state, and received no drug treatment, the improved regeneration in this group can be attributed to an altered immune response. This is still indirect evidence in support of our original hypothesis (Feringa et al., 1973, 1975), but other nonspecific effects of weekly injection of CNS material which might result in improved axonal regeneration have not been described.

To date we have been as frustrated as others (Willenborg et al., 1977) in our effort to identify a specific measure of immune reactivity which is present after CNS injury and is specifically altered by those treatments which we have shown increased axonal regeneration in the CNS. Such an immune response need not be cell-mediated. Others have shown both cell-mediated (Sheremata et al., 1976) and humoral immunological responses (Bornstein, 1963; Bowen, 1968; Paterson and
Hanson, 1969, Caspary and Chambers, 1970; Bornstein and Iwanawi, 1971; Prochazka et al., 1971; Clifton et al., 1976) after CNS injury. The CNS contains a variety of antigens. Many types of immunological responses are possible. Even with the large number of tests now available, it is not always possible to characterise fully the nature of a given immunological response.

This study adds to the indirect evidence that an immunological response to CNS tissue in some way interferes with central axonal regeneration.

We would like to express our appreciation to Ms Linda Lee Austin for technical assistance and to Mr Robert McKnight for assistance with art work and photography. Preliminary data from this research were presented at a poster session of the Society for Neuroscience, November 1977, Anaheim, California. The work was supported by research funds from the Veterans Administration, and funds from the University of Michigan Medical Center Fund for Computing, the Gelston Fund, and the Development Fund of the Department of Pathology.

\section{References}

Bornstein, M. D. (1963). A tissue-culture approach to demyelinative disorders. National Cancer Institute Monograph, 11, 197-211.

Bornstein, M. D., and Iwanawi, H. (1971). Experimental allergic encephalomyelitis: demyelinating activity of serum and sensitized lymph node cells on cultured nerve tissues. Journal of Neuropathology and Experimental Neurology, 30, 240-248.

Bowen, F. P. (1968). Immunologic reactions after cortical lesions in rabbits. Archives of Neurology (Chicago), 19, 398-402.

Caspary, E. A., and Chambers, M. E. (1970). Antibody to encephalitogenic basic protein in multiple sclerosis and other neurological disease as measured by immune adherence. European Neurology, 3, 206210.

Claman, H. N., and Bronsky, E. A. (1965). Inhibition of antibody production and acquired immunologic tolerance by actinomycin. Journal of Immunology, 95, 718-721.

Clifton, G. L., Coggeshall, R. E., Vance, W. H., and Willis, W. D. (1976). Receptive fields of unmyelinated ventral root afferent fibres in the cat. Journal of Physiology, 256, 573-600.

Eitzman, D. V., and Smith, R. T. (1959). Antibody response to heterologous protein in rabbits of varying maturity. Proceedings of the Society for Experimental Biology and Medicine, 102, 529-531.

Feringa, E. R., Gurden, G., Strodel, W., Chandler, W., and Knake, J. (1973). Descending spinal motor tract regeneration after spinal cord transection. Neurology (Minneapclis), 23, 599-608. 
Feringa, E. R., Wendt, J., and Johnson, R. D. (1974). Immunosuppressive treatment to enhance spinal cord regeneration in rats. Neurology (Minneapolis), 24, 287-293.

Feringa, E. R., Johnson, R. D., and Wendt, J. (1975). Spinal cord regeneration in rats after immunosuppressive treatment. Archives of Neurology (Chicago), 32, 676-683.

Feringa, E. R., Shuer, L. M., Vahlsing, H. L., and Davis, S. W. (1977). Regeneration of corticospinal axons in the rat. Annals of Neurology, 2, 315-321.

Feringa, E. R., Davis, S. W., Vahlsing, H. L., and Shuer, L. M. (1978). Fink-Heimer/Nauta demonstration of regenerating axons in the rat spinal cord. Archives of Neurology (Chicago), 35, 522526.

Hicks, S. P., and D'Amato, C. J. (1975). Motorsensory cortex-corticospinal system and developing locomotion and placing in rats. American Journal of Anatomy, 143, 1-42.

Hopkins, A. P., and Lambert, E. H. (1972). Conduction in regenerating unmyelinated fibres. Brain, 95, 213-222.

LaVail, J. H. (1975). The retrograde transport method. Federation Proceedings, 34, 1618-1624.

Paterson, P. Y. (1958). Studies of immunological tolerance to nervous tissue in rats. Annals of the New York Academy of Sciences, 73, 811-813.
Paterson, P. Y., and Hanson, M. A. (1969). Studies of cyclophosphamide suppression of experimental allergic encephalomyelitis in Wistar rats. Journal of Immunology, 103, 795-803.

Prochazka, W., Voltnerova, M., and Stefan, J. (1971). Studies of immunologic reactions after brain injury in man. International Surgery, 55, 322-326.

Sheremata, W. J., Cosgrove, B. R., and Eylar, E. H. (1976). Multiple sclerosis and cell-mediated hypersensitivity to myelin A1 protein. Journal of the Neurological Sciences, 27, 413-425.

Smith, R. T., and Bridges, R. A. (1958). Immunological unresponsiveness in rabbits produced by neonatal injection of defined antigens. Journal of Experimental Medicine, 108, 227-250.

Vahlsing, H. L., Feringa, E. R., and Hirschl, R. B (1978). Axonal transport of tritiated proline in the corticospinal tract of the rat spinal cord. Neuroscience Abstracts, 4, 38 .

Weigle, W. O. (1972). Circumventing graft rejection. In Transplantation, chapter 6, pp. 174-176. Edited by J. S. Najarian and R. L. Simmons. Lea and Febiger: Philadelphia.

Weigle, W. O. (1973). Immunologic unresponsiveness. Advances in Immunology, 16, 61-122.

Willenborg, D. O., Staten, E. A., and Eidelberg, E. (1977). Studies on cell-mediated hypersensitivity to neural antigens after experimental spinal cord injury. Experimental Neurology, 54, 383-392. 